\title{
Surgery for Combined Hamartoma of the Retina and Retinal Pigment Epithelium
}

\author{
Charlotte Maria van der Sommen \\ Saskia Helena Margaretha van Romunde Koen van Overdam \\ Department of Vitreoretinal Surgery, The Rotterdam Eye Hospital, Rotterdam, \\ The Netherlands
}

\section{Keywords}

Combined hamartoma of the retina and retinal pigment epithelium - CHRRPE .

Vasoproliferative tumor - Vitreoschisis-induced vitreous cortex remnants · VCR · Vitreoretinal surgery

\begin{abstract}
There is no consensus on whether and when surgical treatment is indicated for combined hamartoma of the retina and retinal pigment epithelium (CHRRPE). We aim to discuss the benefits of surgical intervention and techniques that may improve the outcome. A 24-yearold man experienced progressive visual loss for 6 months in his left eye due to CHRRPE. At presentation, visual acuity was 1.3 LogMAR and fundoscopy revealed extensive tractional preand epiretinal membranes, subretinal exudation, and a vasoproliferative tumor in the inferior periphery. A complete vitrectomy was performed, while paying special attention to vitreous shaving at the vitreous base and removal of vitreoschisis-induced vitreous cortex remnants (VCR) from the retinal surface posterior to the vitreous base. Tractional membranes and internal limiting membrane were peeled, and the vasoproliferative tumor was excised. Silicone oil tamponade was removed 11 weeks after surgery. No intra- or postoperative complications occurred. Visual acuity improved to 0.8 LogMAR and remained stable for 48 -month followup. Vitreoretinal surgery can prevent complications that occur with CHRRPE. In addition, visual function may improve even if the initial visual acuity is low. Timely and complete vitrectomy with extensive membranectomy and detection and removal of VCR is recommended to avoid complications in challenging CHRRPE.
\end{abstract}




\section{Introduction}

Combined hamartoma of the retina and retinal pigment epithelium (CHRRPE) is a rare benign solitary tumor which consists of a predominant tissue subtype located at the optic disc, juxtapapillary region, and posterior pole. It usually presents at a young age and causes significant visual loss depending on its location [1]. Progressive visual loss can occur due to tumor growth and complications associated with the tumor, such as tractional membrane formation.

The typical presentation on optical coherence tomography (OCT) imaging is inward retinal traction without deep retinal disturbances (known as mini-peaks), and inner retinal disorganization (maxi-peaks) limited to the outer plexiform layer (omega sign) [2]. Retinoschisis, development of choroidal or retinal neovascularization, intraretinal and vitreous hemorrhages, retinal tears, and retinal detachments have also been described [3-5].

Surgical intervention to halt or prevent these sequelae is arguable due to the variable outcomes of vitrectomy with membrane peeling, especially when the initial visual acuity is low $[6,7]$. This report aims to discuss the benefits of surgical intervention and techniques that may improve the outcome.

\section{Case Report}

In October 2016, a 24-year-old man was referred to our institution for a second opinion on progressive visual loss for 6 months in his left eye due to CHRRPE with tractional membranes, subretinal exudation, and a vasoproliferative tumor. There was no history of systemic manifestations associated with CHRRPE such as neurofibromatosis type 1 or 2 .

Two years earlier, a best-corrected visual acuity (BCVA) of 0.0 LogMAR in both eyes was measured by an optician. On presentation in our institution, BCVA was 0.0 LogMAR in the right eye and 1.3 LogMAR in the left eye. The intraocular pressure was normal. Slit-lamp examination was unremarkable. Left fundoscopy revealed extensive pre- and epiretinal membranes with traction in the papillomacular area and tortuous blood vessels. There were pigment changes, subretinal exudation, and a large $(>5 \mathrm{~mm})$ vasoproliferative tumor located in the inferior periphery (shown in Fig. 1).

The OCT images showed a pronounced folded distortion of all retinal layers in the macular area causing the "maxi peak" configuration. Central macular thickness was $996 \mu \mathrm{m}$ due to tractional pre- and epiretinal fibrosis. Fluorescence angiography revealed blockage of choroidal flush and progressive hyperfluorescence due to the tractional membranes. There were no signs of vasculitis or secondary neovascularization other than the vasoproliferative tumor. The fellow eye showed no abnormalities.

\section{Surgical Intervention}

A complete 23-gauge vitrectomy, including induction of a posterior vitreous detachment and vitreous shaving with indentation at the vitreous base, was performed by the last author. Special attention was paid to the detection and removal of vitreoschisis-induced vitreous cortex remnants (VCR) over the retinal surface posterior to the vitreous base. Visualization of vitreous and VCR was enhanced by triamcinolone acetonide $(20 \mathrm{mg} / \mathrm{mL}$; Kenacort-A, Bristol-Myers Squibb, New York, USA). Tractional membranes and internal limiting membrane (ILM) were extensively peeled, with the use of infracyanine green $(2.5 \mathrm{mg} / \mathrm{mL}$; SERB Laboratories, Paris, France) for staining of the ILM.

Before excision of the vasoproliferative tumor with the vitrectome, 360-degree endolaser photocoagulation was performed at the vitreous base, followed by endolaser and

\section{Karger's}



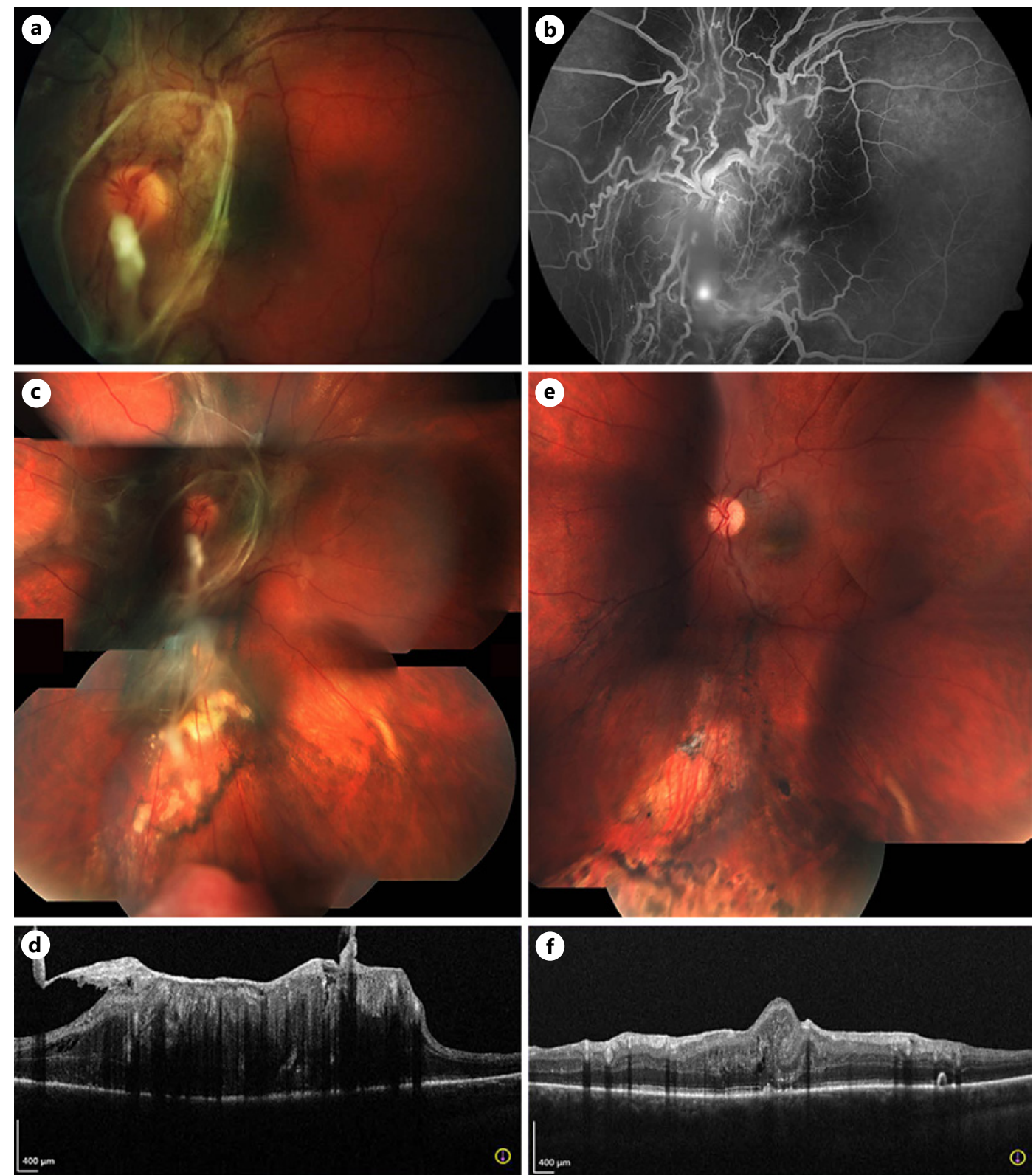

Fig. 1. Pre- and postoperative imaging of a 24-year-old man who underwent vitrectomy for CHRRPE with extensive tractional pre- and epiretinal membranes, subretinal exudation, and a vasoproliferative tumor. Four months preoperatively: extensive pre- and epiretinal membranes with traction in the papillomacular area (A), with blocking of the choroidal flush, tortuous vessels, and progressive hyperfluorescence on fluorescein angiography (B). One month preoperatively: increased retinal traction, pigment changes, subretinal exudation, and a vasoproliferative tumor in the inferior periphery (C), with mini-peak and maxi-peak configurations, and increased retinal thickness on OCT(D). Thirty-eight months postoperatively: significant improvement in retinal anatomy after complete vitrectomy with extensive removal of tractional membranes, vitreoschisis-induced VCR, and the vasoproliferative tumor (E), with decreased macular thickness in the presence of a remaining macular fold on OCT (F). BCVA improved from 1.3 LogMAR to 0.8 LogMAR. CHRRPE, combined hamartoma of the retina and retinal pigment epithelium; VCR, vitreous cortex remnant; OCT, optical coherence tomography; BCVA, best-corrected visual acuity. 
endodiathermy coagulation around the tumor. After fluid-air exchange, the vitreous cavity was filled with 5,000 cs silicone oil. An intraocular injection of $0.05 \mathrm{~mL}$ bevacizumab $(1.25$ $\mathrm{mg} / 0.05 \mathrm{~mL}$; Avastin ${ }^{\circledR}$, Roche Pharma AG, Grenzach- Wyhlen, Germany) and a subconjunctival injection of $1.0 \mathrm{~mL}$ betamethasone ( $4 \mathrm{mg} / \mathrm{mL}$; Celestone, Merck \& Co, Kenilworth, USA) were administered at the end of surgery. No intraoperative complications occurred. A compilation of the surgery can be seen in the video.

\section{Outcome}

There were no complications 1 day postoperatively, that is, no hemorrhage, inflammation, or retinal detachment, and a normal intraocular pressure. Two months after surgery, BCVA improved to 1.0 LogMAR. Eleven weeks after surgery the silicone oil was removed. Follow-up was uneventful, the visual acuity remained stable and OCT images showed further decrease of macular folds and retinal thickness. On the last visit, 48 months postoperatively, BCVA was 0.8 LogMAR with minimal posterior subcapsular opacification of the lens, otherwise clear media and attached retina, without proliferative vitreoretinopathy (PVR), traction, or exudation. The central macular thickness decreased to $640 \mu \mathrm{m}$ in the presence of a remaining macular fold (shown in Fig. 1).

\section{Discussion}

Since the first scientific documentation of CHRRPE [8], there have only been several small studies describing results of vitreoretinal surgery. In 1985 McDonald et al. [6] reported poor surgical outcome in 2 patients with CHRRPE and secondary long-standing low visual acuity and marked cystoid macular edema. In the following years, others showed more promising outcomes when removing epiretinal membranes in CHRRPE $[9,10]$. They recommended consideration of early membranectomy in patients with relatively good visual acuity. They stated that even in long-standing low BCVA ( $\leq 20 / 100$ Snellen) and a decrease below 20/400 Snellen, surgery might be beneficial. Since the publication of these small case studies, there have been great improvements in vitreoretinal instrumentation, techniques, and adjuvants, including improved fluidics control, small-gauge instruments, better internal light sources, and development of new dyes. This enables better surgical outcomes with less complications.

Even with these technical improvements, the decision if and when to perform vitreoretinal surgery is still challenging. Due to macular involvement of CHRRPE and/or longstanding macular edema with tractional membranes, visual recovery may be limited. In case of good visual acuity, watchful waiting using regular OCT assessment is usually appropriate. In this way, retinal- and vascular changes can be detected at an early stage and timely treatment can be considered when visual acuity and/or retinal morphology deteriorates [11]. When a secondary CNV with exudation is the main reason for visual decline, photodynamic therapy (PDT) or anti-vascular endothelial growth factor could be considered [12]. In our case, we decided to perform surgery without these modalities because of the rapid and progressive visual deterioration due to extensive retinal traction in the absence of a secondary CNV.

As a result of the surgery, we achieved a stable situation with slight improvement in visual acuity. Retinal folds, caused by the tractional membranes, flattened and no recurrence of tractional membranes, exudation, or a vasoproliferative tumor was recorded during 48-month follow-up. It is to be expected that an even better result could be achieved if surgery was performed at an earlier stage, with as a result less impairment of vision and anatomy.

To reduce the risk of postoperative PVR development and subsequent retinal detachment, we recommend the following: (1) complete vitrectomy with the use of triamcinolone acetonide

\section{Karger'}


for vitreous visualization, (2) shaving with indentation at the vitreous base, (3) extensive peeling of tractional membranes and ILM, and (4) detection and removal of VCR. In addition, it is essential to prevent iatrogenic retinal tears, hemorrhages, and inflammation.

Because VCR may act as a scaffold for fibrocellular proliferation, detection and removal of VCR from the retinal surface can reduce the risk of postoperative PVR-related retinal detachment [13]. We used end-gripping forceps and a silicone tipped cannula for VCR removal in this case. Removal of VCR with instruments that are not purpose-built can be difficult and time-consuming. The underlying retina can be easily damaged. A recently described new technique, vitreous wiping, could be useful for a more efficient and safer way to remove VCR [14].

The vasoproliferative tumor was successfully treated with lesion excision. The lesion appeared to be too large for effective and safe treatment with laser- or cryotherapy. Cryotherapy, in this clinical situation, may have led to an increased PVR risk due to wide tissue destruction, intense inflammatory reaction, vitreous hemorrhage, and increase in subretinal fluid.

To prevent intra- and postoperative hemorrhage after retinal vascular tumor resection, and thereby reducing the subsequent risk of PVR, effective coagulation around the excision site is important [15]. A recently developed intra-ocular bipolar diathermy forceps may assist the vitreoretinal surgeon in both membrane peeling and more effective blood vessel coagulation [16]. Although a gas tamponade would probably suffice, silicone oil was used in order to have a longer tamponade at the excision site and to prevent diffuse hemorrhage in the vitreous cavity in case of insufficient coagulation.

Preoperative anti-vascular endothelial growth factor and/or PDT could also be considered for the treatment of retinal vasoproliferative tumors and may reduce the risk of intra- or postoperative bleeding after tumor excision [17]. However, PDT is difficult to apply to a tumor in the far periphery, and these treatment modalities could potentially lead to an increase in fibrosis and retinal traction.

\section{Conclusions}

Significant advances in vitreoretinal surgery with promising new techniques and instrumentation allow surgery to be performed at an earlier stage with a reduced risk of intra- and postoperative complications and an increased chance of improving visual function and retinal anatomy in patients with CHRRPE, even in cases of initially poor visual acuity. Timely, complete vitrectomy with detection and removal of VCR is recommended to avoid complications in challenging cases such as CHRRPE.

\section{Statement of Ethics}

This case study has been conducted according to the tenets of the Declaration of Helsinki. Ethical approval was not required for this study in accordance with local guidelines. The patient gave written informed consent to publish his anonymized medical data, fundus images, and OCT scans for this case report.

\section{Conflict of Interest Statement}

Van Overdam has patents on vitreous wipe and intra-ocular bipolar diathermy forceps. The other authors have no conflicts of interest to declare. 
van der Sommen et al.: Surgery for CHRRPE

\author{
Funding Sources
}

There was no funding or grant support for this study.

\title{
Author Contributions
}

C. van der Sommen contributed to acquisition of the data, drafting of the work, and final approval. S. van Romunde: interpretation of data, revising the work critically, and final approval. K. van Overdam: design of the work, interpretation of data, revising the work critically, and final approval.

\section{Data Availability Statement}

All data generated or analyzed during this study are included in this article and its online suppl. material files. Further enquiries can be directed to the corresponding author.

\section{References}

1 Shields CL, Thangappan A, Hartzell K, Valente P, Pirodini C, Shields JA. Combined hamartoma of the retina and retinal pigment epithelium in 77 consecutive patients: visual outcome based on macular versus extramacular tumor location. Ophthalmology. 2008;115:2246-52.

2 Gupta R, Fung AT, Lupidi M, Pappuru RR, Nayak S, Sahoo NK, et al. Peripapillary versus macular combined hamartoma of the retina and retinal pigment epithelium: imaging characteristics. Am J Ophthalmol. 2019; 200(2):263-9.

3 Huot CS, Desai KB, Shah VA. Spectral domain optical coherence tomography of combined hamartoma of the retina and retinal pigment epithelium. Ophthalmic Surg Lasers Imaging. 2009;40:322-4.

4 Theodossiadis PG, Panagiotidis DN, Baltatzis SG, Georgopoulos GT, Moschos MN. Combined hamartoma of the sensory retina and retinal pigment epithelium involving the optic disk associated with choroidal neovascularization. Retina. 2001;21(3):267-70.

5 Wang CL, Brucker AJ. Vitreous hemorrhage secondary to juxtapapillary vascular hamartoma of the retina. Retina. 1984;4(1):44-7.

6 McDonald HR, Abrams GW, Burke JM, Neuwirth J. Clinicopathologic results of vitreous surgery for epiretinal membranes in patients with combined retinal and retinal pigment epithelial hamartomas. Am J Ophthalmol. 1985;100(6):806-13.

7 Schachat AP, Shields JA, Fine SL, Sanborn GE, Weingeist TA, Valenzuela RE, et al. Combined hamartomas of the retina and retinal pigment epithelium. Ophthalmology. 1984;91(12):1609-15.

8 Gass JD. An unusual hamartoma of the pigment epithelium and retina simulating choroidal melanoma and retinoblastoma. Trans Am Ophthalmol Soc. 1973;71:171-5.

9 Sappenfield DL, Gitter KA. Surgical intervention for combined retinal-retinal pigment epithelial hamartoma. Retina. 1990;10(2):119-24.

10 Bruè C, Saitta A, Nicolai M, Mariotti C, Giovannini A. Epiretinal membrane surgery for combined hamartoma of the retina and retinal pigment epithelium: role of multimodal analysis. Clin Ophthalmol. 2013;7:179-84.

11 Zang X, Dong F, Dai R, Yu W. Surgical management of epiretinal membrane in combined hamartomas of the retina and retinal pigment epithelium. Retina. 2010;30(2):305-9.

12 Mennel S, Hausmann N, Meyer CH, Peter S. Photodynamic therapy for exudative hamartoma in tuberous sclerosis. Arch Ophthalmol. 2006;124(4):597-9.

13 van Overdam K. Vitreoschisis-induced vitreous cortex remnants: missing link in proliferative vitreoretinopathy. Acta Ophthalmol. 2020;98(2):e261-2.

14 van Overdam KA, van Etten PG, van Meurs JC, Manning SS. Vitreous Wiping, a new technique for removal of vitreous cortex remnants during vitrectomy. Acta Ophthalmol. 2019;97(5):e747-52.

15 van Overdam KA, Missotten T, Kilic E, Spielberg LH. Early surgical treatment of retinal hemangioblastomas. Acta Ophthalmol. 2017;95(1):97-102.

16 van Overdam KA, Kilic E, Verdijk RM, Manning S. Intra-ocular diathermy forceps. Acta Ophthalmol. 2018; 96(4):420-2.

17 Huang YM, Chen SJ. Clinical characters and treatments of retinal vasoproliferative tumors. Taiwan J Ophthalmol. 2016;6(2):85-8. 